\title{
Optimization of Mn doping in group-IV-based dilute magnetic semiconductors by electronic codopants
}

\section{Citation}

Chen, Hua, Wenguang Zhu, Efthimios Kaxiras, and Zhenyu Zhang. 2009. "Optimization of Mn Doping in Group-IV-Based Dilute Magnetic Semiconductors by Electronic Codopants." Physical Review B 79 (23). https://doi.org/10.1103/physrevb.79.235202.

\section{Permanent link}

http://nrs.harvard.edu/urn-3:HUL.InstRepos:41384091

\section{Terms of Use}

This article was downloaded from Harvard University's DASH repository, and is made available under the terms and conditions applicable to Open Access Policy Articles, as set forth at http:// nrs.harvard.edu/urn-3:HUL.InstRepos:dash.current.terms-of-use\#OAP

\section{Share Your Story}

The Harvard community has made this article openly available.

Please share how this access benefits you. Submit a story.

Accessibility 


\title{
Optimization of Mn Doping in Group-IV-based Dilute Magnetic Semiconductors by Electronic Co-dopants
}

\author{
Hua Chen and Wenguang Zhu \\ Department of Physics and Astronomy, University of Tennessee, Knoxville, TN 37996 and \\ Materials Science and Technology Division, Oak Ridge National Laboratory, Oak Ridge, TN 37831 \\ Efthimios Kaxiras \\ Department of Physics and School of Engineering and Applied Sciences, Harvard University, Cambridge, MA 02138 \\ Zhenyu Zhang \\ Materials Science and Technology Division, Oak Ridge National Laboratory, Oak Ridge, TN 37831 and \\ Department of Physics and Astronomy, University of Tennessee, Knoxville, TN 37996
}

(Dated: May 3, 2019)

\begin{abstract}
The percentage of substitutional doping of magnetic atoms (Mn) in group-IV-based dilute magnetic semiconductors (DMS) can be increased by co-doping with another conventional electronic dopant (e-dopant), as demonstrated from first-principles calculations recently [Zhu et al., Phys. Rev. Lett. 100, 027205 (2008)]. Here, we report extensive theoretical investigations of the kinetic and thermodynamic characteristics of several co-doped systems including bulk $\mathrm{Si}$ and Ge as hosts and various group-III and group-V e-dopants. The main findings are as follows: The $n$ - $p$ pairing of $n$-type e-dopants with $p$-type substitutional $\mathrm{Mn}$ is energetically stable in bulk Ge and $\mathrm{Si}$. Mn atoms move from interstitial sites to substitutional sites easier (with lower kinetic barriers) in the presence of a neighboring $n$-type e-dopant. Magnetic coupling between two Mn atoms in bulk Ge oscillates between positive (ferromagnetic) and negative (antiferromagnetic) values with increasing $\mathrm{Mn}-\mathrm{Mn}$ distance, but in $\mathrm{Mn} / \mathrm{As}$ co-doped Ge the coupling parameter remains positive at all distances beyond nearest-neighbors and this qualitative difference does not change with the doping level. For $\mathrm{Mn}$ doped $\mathrm{Si}$, all coupling values except for the nearest neighbor one are positive and do not change much upon co-doping. We find an unconventional magnetic anisotropy in the co-doped system, that is, the dependence of magnetic coupling on the relative positions of the magnetic ions and their neighboring e-dopants. We map the calculated magnetic coupling to a classical Heisenberg model and employ Monte Carlo simulations to estimate the Curie temperature $\left(\mathrm{T}_{c}\right)$. We find that in $\mathrm{Mn}$ doped Ge no ferromagnetic order exists for Mn concentrations ranging from $3.13 \%$ to $6 \%$. Instead, a spin-glass phase transition occurs at $\sim 5 \mathrm{~K}$ at $5 \% \mathrm{Mn}$ doping. For Mn/As co-doped Ge, $\mathrm{T}_{c}$ increases nearly linearly with the Mn concentration and reaches $264 \mathrm{~K}$ at $5 \% \mathrm{Mn}$ doping.
\end{abstract}

PACS numbers: 75.50.Pp, 66.30.Jt, 75.30.Hx

\section{INTRODUCTION}

Dilute magnetic semiconductors (DMS) have attracted much interest in the condensed matter community not only because of their promising application in spintronic devices $\sqrt{12}$, but also because of the many new and important theoretical issues which arise from the study of this unique class of disordered magnetic system $3 / 4|5| 6 \mid 7$. As for specific materials, besides the most extensively studied (III,Mn)V systems $\frac{618}{\text {, }}$ Mn doped group-IV semiconductors such as $\mathrm{Ge}$ and $\mathrm{Si}$ also show promise for real applications $s^{9|10| 11|12| 13|14| 15}$. In order to realize this promise, a Curie temperature comparable to room temperature or higher is required. Both theory and experiment indicate that the Curie temperature of the abovementioned materials is exceptionally sensitive to the ratio of interstitial to substitutional $\mathrm{Mn}$ atoms 716 16/17/19]. In (III,Mn)V as well as (Mn,IV) systems, substitutional Mn atoms act as acceptors and provide holes which, according to current understanding, are the mediator of magnetic interactions between magnetic moments in these materials. Interstitial $\mathrm{Mn}$ atoms $\sqrt{19}$ are identified to be donors, and tend to compensate the holes and magnetic moments induced by the substitutional $\mathrm{Mn}[$. Furthermore, though annealing is an effective way to decrease the percentage of interstitial Mn while keeping the homogeneity in $(\mathrm{Ga}, \mathrm{Mn}) \mathrm{As},{ }^{20 \mid 21}$ it is less useful for $\mathrm{Mn}_{x} \mathrm{Ge}_{1-x}$ and $\mathrm{Mn}_{x} \mathrm{Si}_{1-x}, 22 \mid 23 \sqrt[2425]{25}$ which makes it very difficult to get high quality samples of these materials using conventional methods.

In our recent work ${ }^{26}$, a novel way to enhance the substitutional doping of $\mathrm{Mn}$ in $\mathrm{Ge}$ and $\mathrm{Si}$ was proposed. In this method, an additional conventional electronic dopant (e-dopant) such as As or P is introduced in the doping process. Using first-principles electronic structure calculations, we were able to show that the co-doping approach can substantially lower the energy of Mn atoms at substitutional sites relative to that at interstitial sites, as well as the energy barrier which the Mn atoms have to overcome in order to be incorporated into substitutional sites. In addition, the assisting e-dopant enhances the magnetic coupling between substitutional Mn atoms. A new type of magnetic anisotropy was also found, which depends on the proximity of the assisting e-dopant to a 
Mn dopant, rather than the direction of magnetic moments relative to the lattice direction of the host or to other moments.

In this paper, we present a detailed ab initio investigation of this novel approach by analyzing the kinetic and thermodynamic issues related to the stability of various dopant-host combinations. We then calculate the magnetic coupling between two Mn atoms in bulk Ge and Si, and find that in Ge, the coupling oscillates between positive (ferromagnetic) and negative (antiferromagnetic) values with the $\mathrm{Mn}-\mathrm{Mn}$ distance. But in $\mathrm{Mn} / \mathrm{As}$ codoped Ge the coupling parameter remains positive at all distances beyond nearest-neighbors, and this qualitative difference does not change with the doping level. For $\mathrm{Mn}$ doped $\mathrm{Si}$, all the couplings except for the nearest neighbor one are positive and do not change much upon co-doping. We also carry out Monte Carlo simulations to obtain the Curie temperatures of the co-doped materials. We find that in $\mathrm{Mn}$ doped Ge no ferromagnetic order exists for Mn concentrations ranging from 3.13\% to $6 \%$. Instead, a spin-glass phase transition occurs at $\sim 5 \mathrm{~K}$ at $5 \% \mathrm{Mn}$ doping. For $\mathrm{Mn} / \mathrm{As}$ co-doped $\mathrm{Ge}, \mathrm{T}_{c}$ increases nearly linearly with the Mn concentration and reaches $264 \mathrm{~K}$ at $5 \% \mathrm{Mn}$ doping.

The paper is organized as follows: In section II we present the computational methodology, including details of our $a b$ initio treatment and the Monte Carlo simulations. The main $a b$ initio results are given in Sec III, where the kinetic and energetic properties of various combinations of host materials ( $\mathrm{Ge}, \mathrm{Si}$ ) and assisting dopants (As, P, Al, Ga) are investigated. Magnetic interactions in As co-doped $\mathrm{Mn}_{x} \mathrm{Ge}_{1-x}$ are discussed in Sec IV] In Sec.V the $a b$ initio results of magnetic coupling are used to find the transition temperature $\mathrm{T}_{c}$ of $\mathrm{Mn} / \mathrm{As}$ co-doped Ge. The discussion and summary are provided in the last two sections.

\section{METHODS}

Our spin-polarized first-principles calculations are carried out using the Vienna ab initio simulation package (VASP) $)^{27}$, a density functional theory approach using the projector augmented wave (PAW) method ${ }^{28129}$ and the generalized gradient approximation (PBE-GGA) ${ }^{30}$ for exchange-correlation. A default plane-wave energy cutoff of $269.9 \mathrm{eV}$ is consistently used in all Mn calculations. These choices produce a bulk Ge and Si lattice constants of $5.78 \AA$ (experimental value ${ }^{31} 5.66 \AA$ ) and 5.47 $\AA$ respectively (experimental valuc ${ }^{32} 5.43 \AA$ ).

In our calculations of the co-doping process the supercell size is chosen to be a $2 \times 2 \times 2$ multiple of the conventional cubic cell of the diamond lattice which contains 8 atoms. Hence, there are 64 atoms in one supercell, and one of them is replaced by an Mn atom, corresponding to $1.56 \% \mathrm{Mn}$ concentration, comparable to what was achieved experimentally 1934135 . Different supercell sizes were used to study the dependence of calculated results on Mn concentration. Specifically, we used a $3 \times 3 \times 3$ supercell, which corresponds to 216 atoms, and with one of them replaced by a $\mathrm{Mn}$ the concentration is $0.46 \%$. In each calculation of the magnetic coupling between $\mathrm{Mn}$ atoms, two $\mathrm{Mn}$ atoms are placed in a $3 \times 3 \times 3$ supercell, corresponding to a $0.926 \% \mathrm{Mn}$ concentration. We also selectively use a $2 \times 2 \times 2$ supercell for the magnetic coupling with two $\mathrm{Mn}$ atoms in the supercell, corresponding to $3.125 \% \mathrm{Mn}$, for comparison. This setup is similar to previous studies of Mn-Mn interactions in pure semiconductors $\$ 36 / 37 / 38 / 39$.

A uniform $4 \times 4 \times 4(2 \times 2 \times 2)$ mesh, including the $\Gamma$ point $(0,0,0)$, is chosen for Brillouin Zone sampling in the $2 \times 2 \times 2(3 \times 3 \times 3)$ supercell. Optimized atomic geometries are obtained when the forces on all the unconstrained atoms are smaller in magnitude than $0.01 \mathrm{eV} / \AA$. The "climbing image Nudged Elastic Band" (NEB) method ${ }^{\sqrt{33}}$ is used to locate the transition state geometries for the calculation of activation energy barriers. Typically four slab replicas between the initial and final geometries are enough to produce a smooth minimum energy path.

For the Monte Carlo simulations we use the Metropolis algorithm $\frac{40}{4}$ and the magnetic energy of the system is calculated using the classical Heisenberg model, in which each magnetic ion is treated as a classical moment and is placed at a randomly chosen site of the supercell. The magnetic coupling parameters are extracted from $a b i n i-$ tio results of the energy difference between parallel and antiparallel spin configurations of two Mn moments at different separations. At each temperature we use 50000 Monte Carlo steps per moment for the system to relax, and calculate the thermal average in the following 50000 steps. To determine the Curie temperature, we adopt the fourth order cumulant crossing method based on finitesize scaling theory proposed by Binder $\underline{40 \mid 41}$. In applying this method we choose three supercell sizes: $8 \times 8 \times 8$, $10 \times 10 \times 10,12 \times 12 \times 12$, and 40 configurations in each case for averaging.

\section{AB INITIO STUDY OF THE CO-DOPING PROCESSES}

\section{A. Study on intrinsic (Mn,IV) without additional dopants}

We first consider the equilibrium structure of a single Mn dopant atom in bulk Si and Ge, and address the difficulty of lowering the percentage of interstitial Mn impurities. A complete understanding of the microscopic doping process requires detailed knowledge of the energetics as well as the kinetics of dopants in the host material16. In fact, an in-depth understanding of the growth kinetics is particularly important, because DMS systems are typically in a metastable state, since they are grown by co-doping the magnetic dopants and host semiconductor atoms using molecular beam epitaxy under nonequilib- 

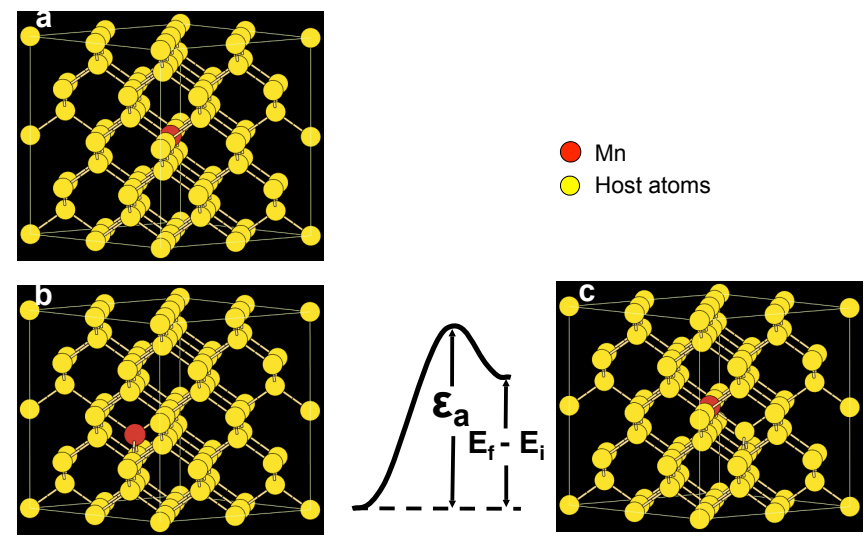

FIG. 1: (color online) Different Mn sites in bulk Si or Ge: (a) Mn at a substitutional site. (b) Mn at an interstitial site. (c) Final state of an interstitial Mn kicking out a neighboring host atom to an interstitial site and occupying the left-behind substitutional one.

rium conditions $9 \sqrt{94342143}$.

To address these issues, we calculate the relative formation energy of a substitutional $\mathrm{Mn}$ (Fig. 1(a)) and interstitial Mn(Fig. 1(b)) atom in Ge and Si separately, which is defined as:

$$
\Delta E_{1}=\left(E_{\text {subst }}+\mu_{\text {host }}\right)-E_{\text {inter }},
$$

where $\mu_{\text {host }}$ is the host material's chemical potential. For $\mathrm{Ge}$, our calculation gives $\Delta E_{1}=-0.63 \mathrm{eV}$. Thus, in Ge the substitutional sites have a relatively lower energy and are preferred by Mn atoms. However, for Si the opposite is true and $\Delta E_{1}=+0.58$. This reversed site preference 44 makes it extremely hard to achieve experimentally even a nominal concentration of substitutional $\mathrm{Mn}$ in silicon.

We next consider kinetic aspects of the Mn doping process. In order to get a high ratio of substitutional to interstitial Mn, the process that an interstitial Mn kicks out a host atom and becomes substitutional must take place more often than the reverse process. Accordingly, we calculate the energy difference between the initial (interstitial, (1)(b)) and final (substitutional, (1)(c)) states of this process: $\Delta E=E_{f}-E_{i}$, and the energy barriers $\varepsilon_{a}$ and $\varepsilon_{a}^{\prime}$ for the reverse process. Our calculation shows that for both Ge and $\mathrm{Si} \Delta E$ is positive $(0.82 \mathrm{eV}$ and 2.03 $\mathrm{eV}$ respectively). This energy cost for the transition from initial to final state defines the lower bound of the activation energy barrier for the exchange process, which must be lower than $\sim 0.8 \mathrm{eV}$ for efficient incorporation ( with a standard attempt frequency $10^{12} \mathrm{sec}^{-1}$ ). Moreover, the actual energy barrier $\varepsilon_{a}$ in either case $(1.12 \mathrm{eV}$ for $\mathrm{Ge}$ and $>2 \mathrm{eV}$ for $\mathrm{Si}$ ) is higher than the barrier of the reverse process, which is calculated as $\varepsilon_{a}^{\prime}=\varepsilon_{a}-\Delta E$, with the latter being lower than $0.8 \mathrm{eV}$, further facilitating the reverse processes. Thus, kinetically $\mathrm{Mn}$ is more stable at interstitial sites rather than at substitutional sites in both Ge or $\mathrm{Si}$. In the following section we will address the issue of doping $\mathrm{Mn}$ together with another $n$ - $(\mathrm{P}, \mathrm{As})$ or $p$-type
$(\mathrm{Al}, \mathrm{Ga})$ conventional e-dopant in order to explore how the assisting dopants influence this site preference both energetically and kinetically.

\section{B. Energetic and kinetic study on the co-doped systems}

Substitutional Mn in Ge is a $p$-type double acceptor ${ }^{45}$. Our proposal for a co-doping mechanism is based on the fact that the electrostatic interaction between a $n$-type and a $p$-type dopant in a semiconductor is attractive because of their different charge states (see below). Thus an $n$-type e-dopant may help to stabilize substitutional Mn atoms.

We start by noting that in Ge or Si there are two kinds of interstitial sites: the hexagonal interstitial site $I_{H}$, which has six nearest neighbors, and the tetrahedral site $I_{T}$ with four nearest neighbors. Using first-principles calculations we find that in $n$-type doped Ge and $\mathrm{Si}$, the energy of a Mn sitting at the $I_{H}$ site is different from that at the $I_{T}$ site. For P, As and Sb doped Ge, the energy differences are $0.14,0.09$ and $0.04 \mathrm{eV}$, respectively, where a positive sign means the $I_{H}$ occupation has lower energy and is preferred. In the case of either $n$ - or $p$ doped $\mathrm{Si}$ as well as $p$-doped Ge, $I_{T}$ is preferred to $I_{H}$. This dopant dependent preference can be qualitatively explained by the local strain effect. Namely, a Mn atom and an $n$-type dopant favor a relatively short bonding distance, which is accommodated by Mn occupying the $I_{H}$ site rather than the $I_{T}$ site in Ge (the $I_{H}$ site has a shorter distance to its nearest neighbors than the $I_{T}$ site). To show that this is indeed the case, we reduce the lattice constant of Ge to the value of $\mathrm{Si}$ and calculate the energy difference again. Then the results show that the preference for $\mathrm{Mn}$ is changed to the $I_{T}$ site, because in this case the distance between the $I_{H} \mathrm{Mn}$ and $n$-type dopant becomes too short (compressive), whereas at the $I_{T}$ site the $\mathrm{Mn} / n$-type dopant bond length is close to its optimal value. We have also checked to confirm that if we increase the lattice constant of $\mathrm{Si}$ to that of Ge, the preference for $\mathrm{Mn}$ is changed to the $I_{H}$ site for the $n$-type doped systems.

In the following we examine two possible kinetic processes of an interstitial Mn atom becoming substitutional. These processes share the same initial state with Mn occupying either the $I_{H}$ or $I_{T}$ sites with a neighboring $n$ type or $p$-type e-dopant. From our calculation of the total energy of a Ge-supercell with an interstitial Mn and a substitutional e-dopant as a function of their separation, shown in Fig. 2, we find that shorter separation is energetically preferred. Thus, the choice of neighboring $\mathrm{Mn} / \mathrm{e}$-dopant pair configuration is reasonable.

In the first process, denoted as Process I, we consider an interstitial Mn directly exchanging position with its substitutional e-dopant neighbor. In the final state, the e-dopant is pushed to an adjacent interstitial site and the Mn atom moves into the substitutional site left behind, 


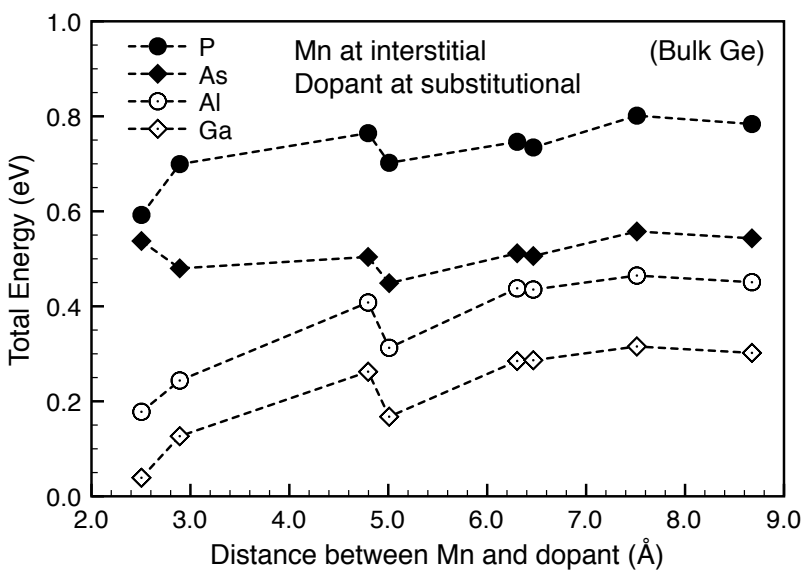

FIG. 2: Calculated relative total energy as a function of the distance between an interstitial $\mathrm{Mn}$ and a substitutional dopant in bulk Ge.

as shown in Fig. 3. Table 1 summarizes the calculated energy differences $\Delta E$ between the final and initial states for $n$-type and $p$-type dopants in Si and Ge. We find that only the $\mathrm{P}$ or As doped Ge (with $\Delta E=0.33 \mathrm{eV}$ and 0.42 $\mathrm{eV}$ respectively) can fulfill the requirement that $\Delta E<0.8$ $\mathrm{eV}$. However, further examination of the activation energy barriers for incorporation in these two cases gives $\varepsilon_{a}=0.88 \mathrm{eV}$ and $0.98 \mathrm{eV}$ respectively, which means this process is unlikely to happen in both cases. Moreover, the reverse processes with $\varepsilon_{a}^{\prime}=0.55 \mathrm{eV}$ and $0.56 \mathrm{eV}$, respectively, are more likely to occur.

Nevertheless, there is one possibility for the Mn atom to stay at the substitutional site, that is, the kicked-out dopant atom diffuses away rapidly so that the reverse process cannot happen. This is ruled out by our calculation of the energy of a Mn/e-dopant pair as a function of their separation, shown in Fig,4, which shows that the e-

\section{Initial states}

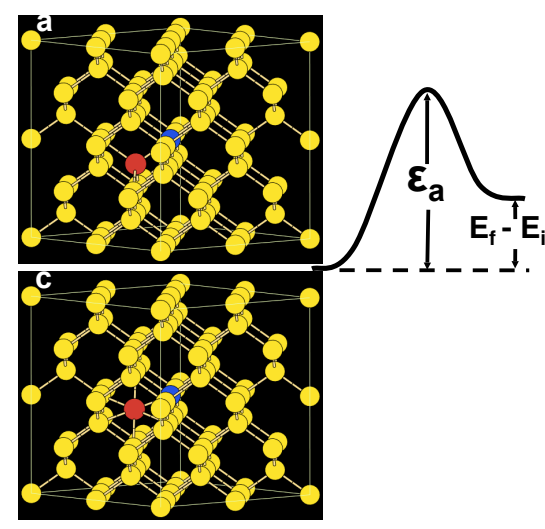

Final states

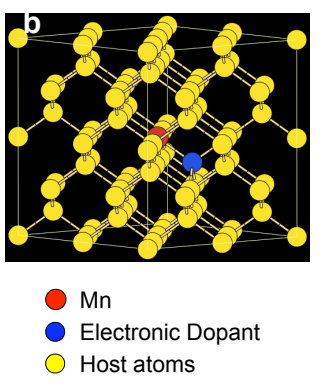

FIG. 3: (color online) Atomic structures and schematic energy profiles for Process I: (a) Initial state with $\mathrm{Mn}$ in the $I_{T}$ position (except for $n$-type doped Ge); (b) Final state; (c) Initial state for $n$-type doped Ge with $\mathrm{Mn}$ at the $I_{H}$ position.

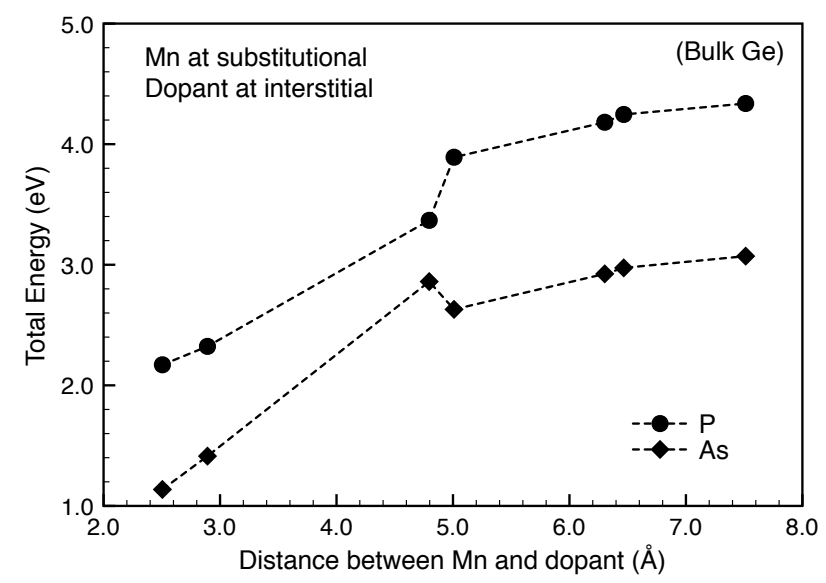

FIG. 4: Calculated relative total energy as a function of the distance between a substitutional $\mathrm{Mn}$ and an interstitial $n$ type dopant in bulk Ge.

dopant cannot diffuse away because the energy increases with increasing separation.

We then consider a different process (Process II), which starts from the same initial configuration as in Process I, but instead of exchanging with the e-dopant, the Mn atom now pushes out a host atom next to the e-dopant to an interstitial site, and then occupies the substitutional site left behind. The final state is shown in Fig. 5, in which the kicked-out interstitial host atom, the substitutional $\mathrm{Mn}$ and the e-dopant are nearly collinear. The calculated $\Delta E$ and $\varepsilon_{a}$ for various $n$-type and $p$-type dopants in $\mathrm{Si}$ and $\mathrm{Ge}$ is also shown in Table I. For $n$-type doped Ge these values are substantially lower than in Process I, and considerably below the threshold value of $0.8 \mathrm{eV}$. For $\mathrm{P}$ and As doped Ge, $\Delta E$ is actually quite low. Furthermore, the activation barriers $\varepsilon_{a}$ for all the three $n$-type dopants is lees than $0.4 \mathrm{eV}$. Qualitatively, this substantial change in the energetic and kinetic characters originates from the electrostatic attraction between the Mn atom, which behaves like a $p$-type dopant, and $n$-type dopants. Therefore, Process II, leading to substitutional Mn atoms proximate to $n$-type dopants, is more likely to happen in reality.

One issue that arises at this stage is whether the final state is thermodynamically stable. To address this question, we calculate the energy difference between interstitial Mn and substitutional Mn defined as

$$
\Delta E_{2}=\left(E_{\text {pair }}+\mu_{\text {host }}\right)-E_{\text {inter }} .
$$

Here $E_{\text {inter }}$ is the total energy of a Mn/e-dopant pair, with the Mn sitting at an interstitial site, while $E_{\text {pair }}$ is that with the Mn occupying a substitutional site. The calculated interstitial-substitutional energy difference $\Delta E_{2}$ is shown in Table II. Compared to the results without $n$-tyep dopants in Sec. IIIA, the substitutional $\mathrm{Mn}$ in Ge becomes much more stable with the neighboring $n$-type dopant. Moreover, the site preference of $\mathrm{Mn}$ in $\mathrm{Si}$ is reversed from interstitial to substitutional. 
TABLE I: Calculated energy differences $\Delta E=E_{f}-E_{i}$ (in eV) between the final and initial states of Process I and Process II, illustrated in Fig.1. $\varepsilon_{a}$ (in eV) is the activation energy for a transition from the initial to final state. Results highlighted in bold correspond to processes for which $\Delta E$ or $\varepsilon_{a}$ or both are $<0.8 \mathrm{eV}$. All the results are for Mn concentration of $1.56 \%$; results for selected cases with Mn concentration of $0.46 \%$ are given in brackets.

\begin{tabular}{|c|c|c|c|c|c|c|c|}
\hline \multicolumn{3}{|c|}{ Bulk Si } & \multicolumn{5}{|c|}{ Bulk Ge } \\
\hline \multirow[t]{2}{*}{$\mathbf{X}$} & \multicolumn{2}{|c|}{$\Delta E=E_{f}-E_{i}$} & \multirow[t]{2}{*}{$\mathbf{X}$} & \multicolumn{2}{|c|}{$\Delta E=E_{f}-E_{i}$} & \multicolumn{2}{|c|}{$\varepsilon_{a}$} \\
\hline & Proc.I & Proc.II & & Proc.I & Proc.II & Proc.I & Proc.II \\
\hline $\mathrm{Si}$ & 2.03 & & Ge & $0.82[1.46]$ & & & \\
\hline $\mathbf{P}$ & 1.46 & 0.89 & $\mathbf{P}$ & $0.33[0.59]$ & $0.03[0.17]$ & 0.88 & 0.34 \\
\hline As & 1.55 & 1.09 & As & $0.42[0.66]$ & $0.05[0.34]$ & 0.98 & 0.25 \\
\hline Al & 1.24 & 2.05 & Al & 0.94 & 1.54 & & \\
\hline $\mathbf{G a}$ & 1.79 & 2.43 & Ga & 1.05 & 1.52 & & \\
\hline
\end{tabular}

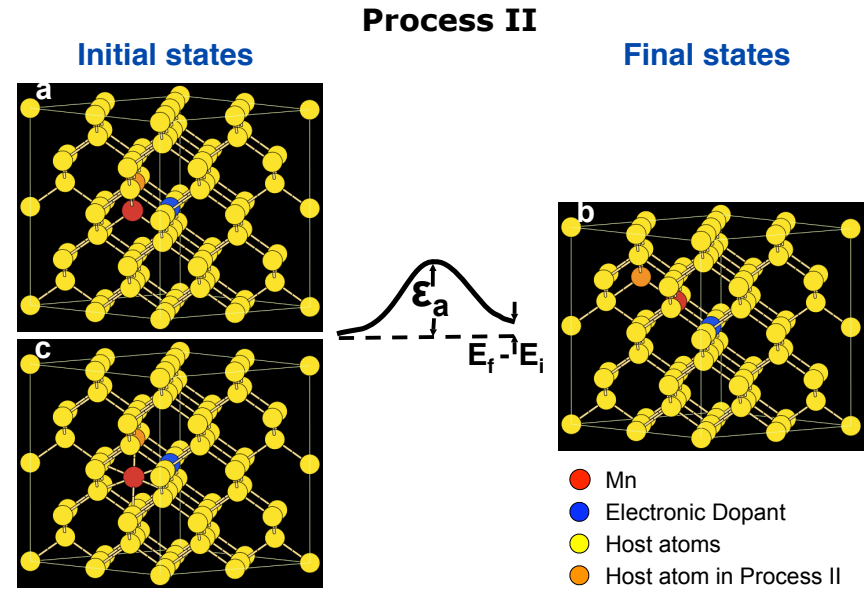

FIG. 5: (color online) Atomic structures and schematic energy profiles of Process II: (a) Initial state with Mn in the $I_{T}$ position (except for $n$-type doped Ge); (b) Final state; (c) Initial state for $n$-type doped Ge, with $\mathrm{Mn}$ at the $I_{T}$ position.

We next calculate the total energy of a Ge-supercell doped by a substitutional Mn/e-dopant pair at different separations. The trend of the total energy with increasing distance between the $\mathrm{Mn}$ atom and the e-dopant is shown in Fig. 6. The interaction between $\mathrm{Mn}$ and edopant is attractive for $n$-type e-dopants (P and $\mathrm{As})$ and repulsive for $p$-type e-dopants ( $\mathrm{Al}$ and $\mathrm{Ga}$ ). This suggests that the picture of electrostatic interaction between $\mathrm{Mn}$ and e-dopants that we proposed at the beginning of this

TABLE II: Relative formation energy of substitutional and interstitial $\mathrm{Mn}$ in the presence of a neighboring substitutional $n$-type dopant, defined as: $\Delta E_{2}=\left(E_{\text {pair }}+\mu_{\text {host }}\right)-E_{\text {inter }}$ (in $\mathrm{eV})$. Negative values indicate higher stability of the substitutional configuration over the interstitial. The relative energy of substitutional and interstitial $\mathrm{Mn}$ in pure Si or Ge are included for comparison.

\begin{tabular}{lccc}
\hline \hline & $\mathrm{P}$ & $\mathrm{As}$ & Undoped \\
\hline $\mathrm{Si}$ & -0.84 & -0.87 & +0.58 \\
$\mathrm{Ge}$ & -1.35 & -1.42 & -0.63 \\
\hline \hline
\end{tabular}

section is valid.

Finally we note that the energy differences between the initial and final states depend on the Mn concentration, as illustrated in Table I. The calculated $\Delta \mathrm{E}$ values at the $0.46 \% \mathrm{Mn}$ concentration are larger than those at the $1.56 \%$ concentration, but for the important cases of $n$-type dopants in Ge, these energy differences are still much lower than the threshold of $\sim 0.8 \mathrm{eV}$. This relatively strong dependence is not due to constant volume calculations, because it is also observed when the supercell volume is fully relaxed. Instead, it is caused by the interaction between the $\mathrm{Mn}$ atoms in adjacent supercells. We stress that the qualitative picture that the $n$-type dopants facilitate substitutional incorporation of $\mathrm{Mn}$ is valid for all the experimentally accessible Mn concentrations considered here.

In short, we have shown that in the presence of a neighboring $n$-type dopant, the substitutional sites are energetically preferred by $\mathrm{Mn}$ atoms to interstitial sites and are kinetically accessible. In the following sections we will turn to study the electronic and magnetic properties of this new $n$ - $p$ co-doped system.

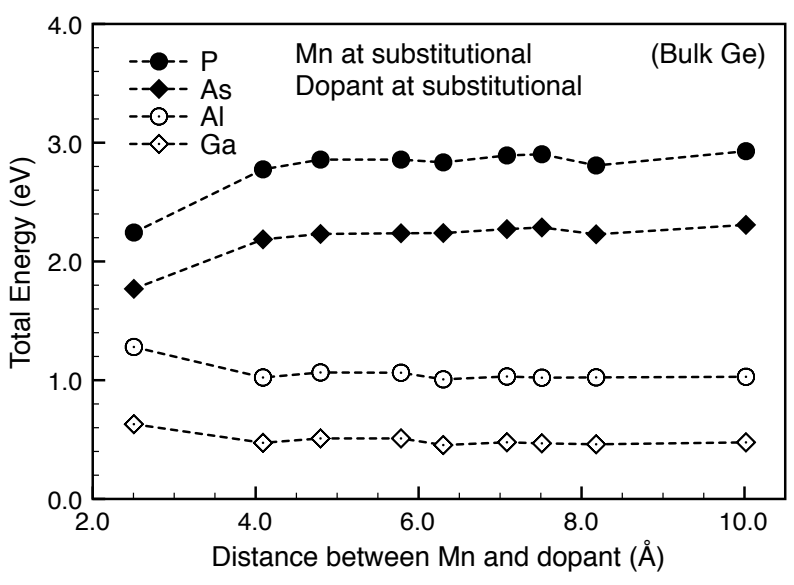

FIG. 6: Relative total energy as a function of the distance between a substitutional $\mathrm{Mn}$ and a substitutional e-dopant in bulk Ge. 


\section{ELECTRONIC AND MAGNETIC PROPERTIES OF THE CO-DOPED SYSTEMS}

\section{A. Electronic structure}

The electronic properties of the Mn/e-dopant co-doped systems are conveniently presented through the calculated density of states (DOS). Fig. 7 and Fig. 8 show the total DOS and local DOS for the substitutional Mn and e-dopants in Ge and Si, respectively. Several important features emerge:

(1) Mn doped Ge or Si are all half metals, regardless of the existence of e-dopants like As or P, which means the value of the total magnetic moment per $\mathrm{Mn}$ atom is integer.

(2) From the figures it can be determined that the moment per $\mathrm{Mn}$ is $3 \mu_{B}$ in pure $\mathrm{Ge}$ or $\mathrm{Si}$, and $4 \mu_{B}$ after co-doping with another e-dopant. The importance of this finding, namely, co-doping can actually increase the magnetic moment of $\mathrm{Mn}$, will be discussed in the next sub-
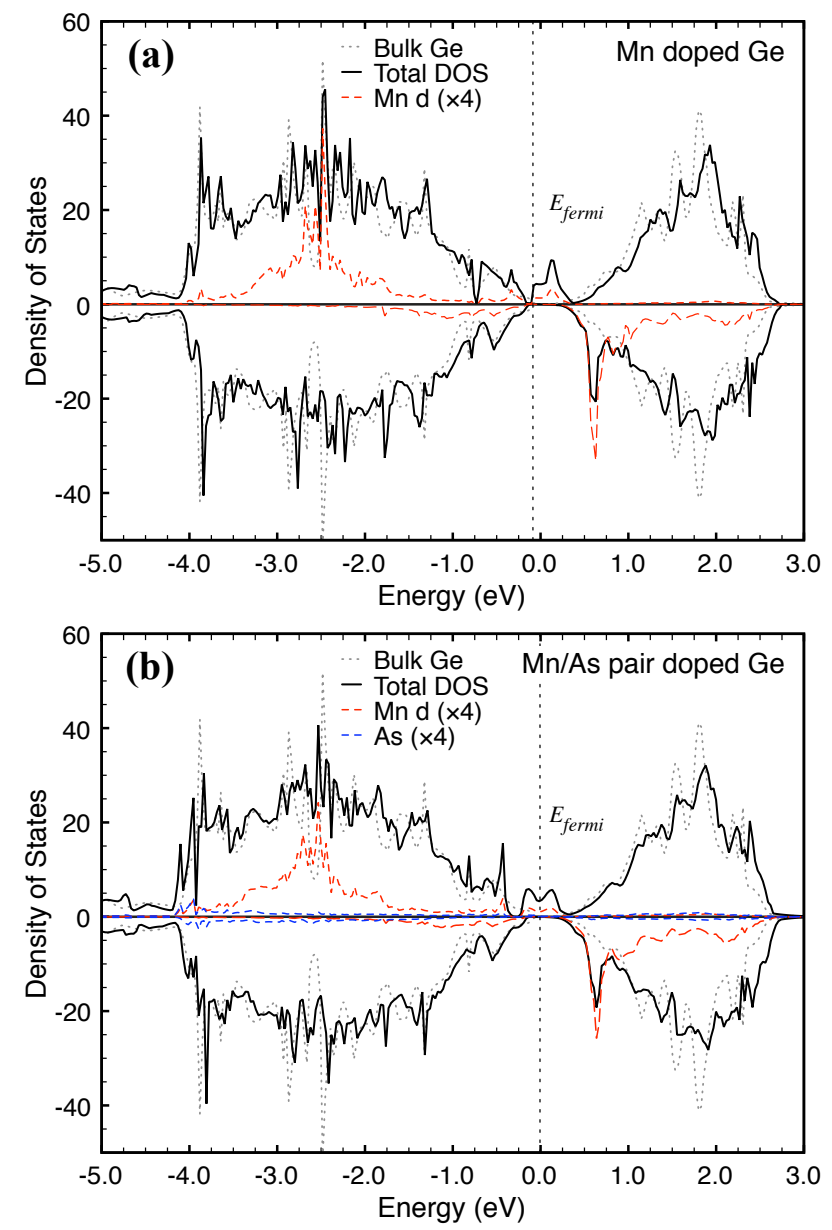

FIG. 7: (color online) The spin-resolved DOS of (a) Mn doped Ge, and (b) a Mn/As pair doped Ge. Projected DOS of $\mathrm{Mn} 3 d$ states and As are given as red and blue dashed lines, respectively. The DOS for bulk Ge is shown for comparison. section.

(3) The local DOS for Mn is broadened to the whole range of the host valence band, indicating that there is strong hybridization between the Mn $d$-state and the valence $p$-state of the host semiconductor.

(4) The local DOS of the additional e-dopant is negligible, meaning that the states it contributes are mostly delocalized, so that its most evident influence on the total DOS is simply to shift the Fermi energy to a higher value, as is expected for a regular non-magnetic dopant.

\section{B. Magnetic properties}

At first sight, the additional $n$-type dopant may negatively influence the strength of the magnetic interaction between Mn atoms, because of the compensation of hole carriers 3 . This argument may not be true for the following reason: We can write the magnetic interaction
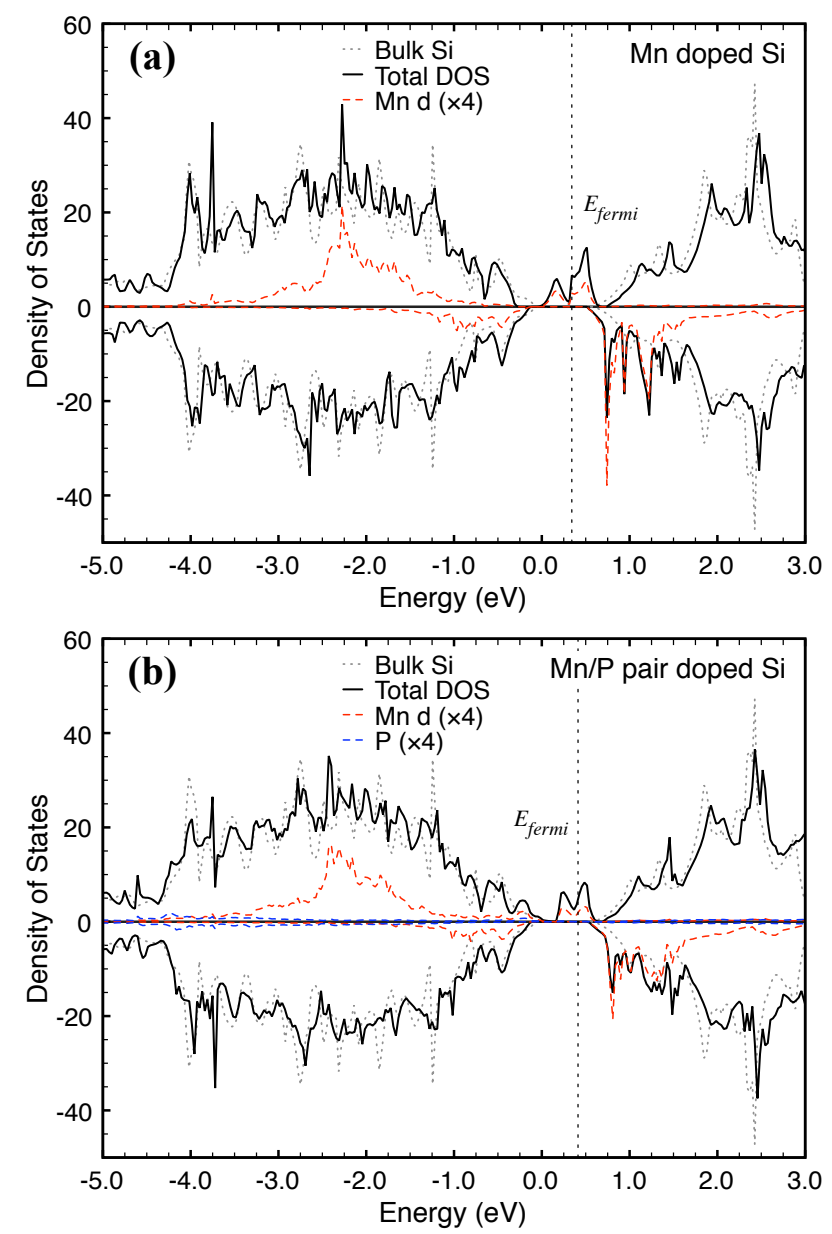

FIG. 8: (color online) The spin-resolved DOS of (a) Mn doped Si, and (b) a Mn/P pair doped Si. Projected DOS of $\mathrm{Mn} 3 d$ states and $\mathrm{P}$ are given as red and blue dashed lines, respectively. The DOS for bulk $\mathrm{Si}$ is shown for comparison. 
energy between two Mn ions as:

$$
E_{M n_{1}-M n_{2}}=J_{e f f} S_{M n_{1}} \cdot S_{M n_{2}}
$$

where $J_{\text {eff }}$ denotes the effective magnetic coupling strength and $S_{M n_{1}}, S_{M n_{2}}$ represent the local magnetic moments associated with the Mn atoms; even if $J_{\text {eff }}$ were to decrease because of the carrier compensation effect, since the influence of the e-dopant on the local moments of $\mathrm{Mn}$ is positive as mentioned in point (2) of the previous subsection, it is still possible that the enhancement of $\mathrm{Mn}$ moments by the e-dopants outweighs its negative influence on $J_{\text {eff }}$.

To check whether this is the case, we first resort to direct $a b$ initio calculation of the magnetic coupling energy of a Mn-Mn pair with different separations, which can be represented by the total energy difference $\Delta E$ between the antiferromagnetic (AFM) and the ferromagnetic $(\mathrm{FM})$ states of the pair $\frac{36 / 37 / 38 / 39}{2}$. In the present case, each $\mathrm{Mn}$ atom has an $n$-type dopant neighbor, which leads to more spatial configurations with the same Mn-Mn distances. To be precise, a substitutional Mn atom has four nearest neighbors, that is, four possible sites for the $n$-type dopant atom, and thus there are 16 possible configurations for a given $\mathrm{Mn}-\mathrm{Mn}$ distance. The number of nonequivalent configurations for each of 12 Mn-Mn separations in the range $2.4-9.5 \AA$ in $\mathrm{Si}(2.5$ $10.0 \AA$ in $\mathrm{Ge})$ is $2,6,16,4,7,16,4,7,16,10,7$ and 5 respectively, with increasing distance.

With these considerations, our results for $\mathrm{Mn} / \mathrm{As}$ codoped $\mathrm{Ge}$ and $\mathrm{Mn} / \mathrm{P}$ co-doped $\mathrm{Si}$ are shown in Fig. 9. We first note that in the case of two Mn atoms in pure Ge ((a) and (b) of Fig. 9), the behavior of the AFMFM energy difference $\Delta \vec{E}$ is oscillatory between positive and negative values as a function of distance (but monotonically decreasing along different directions, see below). In contrast, the average interaction between the two Mn/As pairs in Ge always favors FM coupling except at the nearest-neighbor Mn-Mn distance, and this characteristics does not change with doping level (compare Fig. 9(a) of $3.125 \% \mathrm{Mn}$ and Fig. 9(b) of $0.926 \% \mathrm{Mn}$ ). In the case of $\mathrm{Si}$ as host, Mn atoms favor FM coupling except for nearest neighbor distance, and this feature does not change upon co-doping.

Though usually it is assumed that the hole-mediated magnetic interaction in dilute magnetic semiconductors is RKKY-like, we find that the FM-AFM oscillation displayed in Fig. 9 should not be treated as a manifesta-

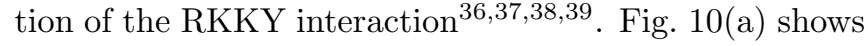
the magnetic coupling between $\mathrm{Mn}$ ions along different lattice directions, which is similar to the design in Mahadevan's work ${ }^{39}$, where it is evident that the oscillatory behavior is replaced by monotonic decrease in magnitude. On the other hand, for the doping levels considered here, the period of RKKY oscillation is much larger than the lattice constant $\frac{46 / 47}{}$, as in the case of GaAs. Thus, the oscillation here is merely due to magnetic anisotropy, rather than a manifestation of RKKYtype interaction. In Fig. 10(b), we plot the coupling along
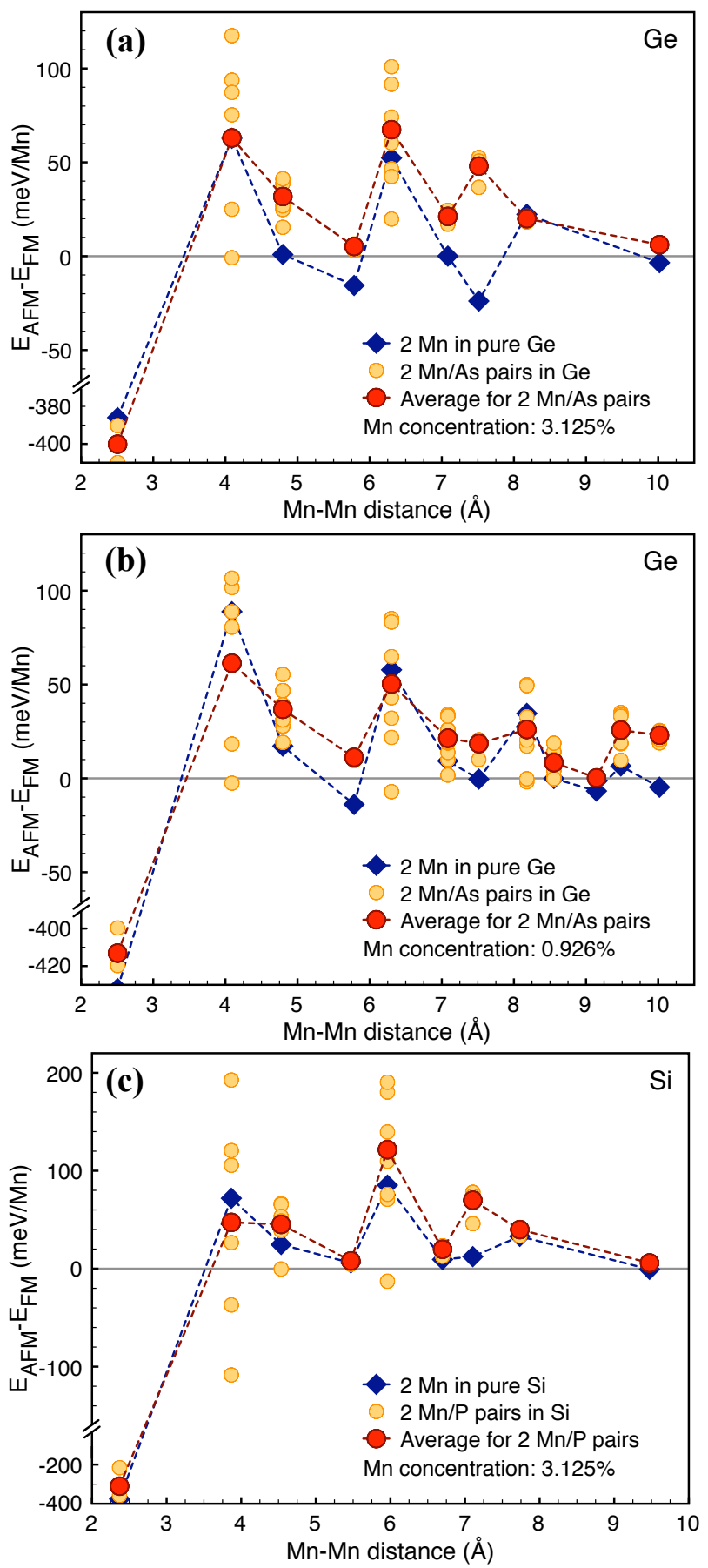

FIG. 9: (color online) Total energy difference between AFM and FM states of two Mn ions versus Mn-Mn separation for two Mn/As pairs doped Ge at (a) 3.125\% and (b) $0.939 \% \mathrm{Mn}$ concentration; (c) for two $\mathrm{Mn} / \mathrm{P}$ pairs doped $\mathrm{Si}$, represented by small orange dots. The large red dots are averages over the small orange dots for a given Mn-Mn distance. For comparison, the results for the systems doped with only two Mn impurities are shown as blue diamonds. 


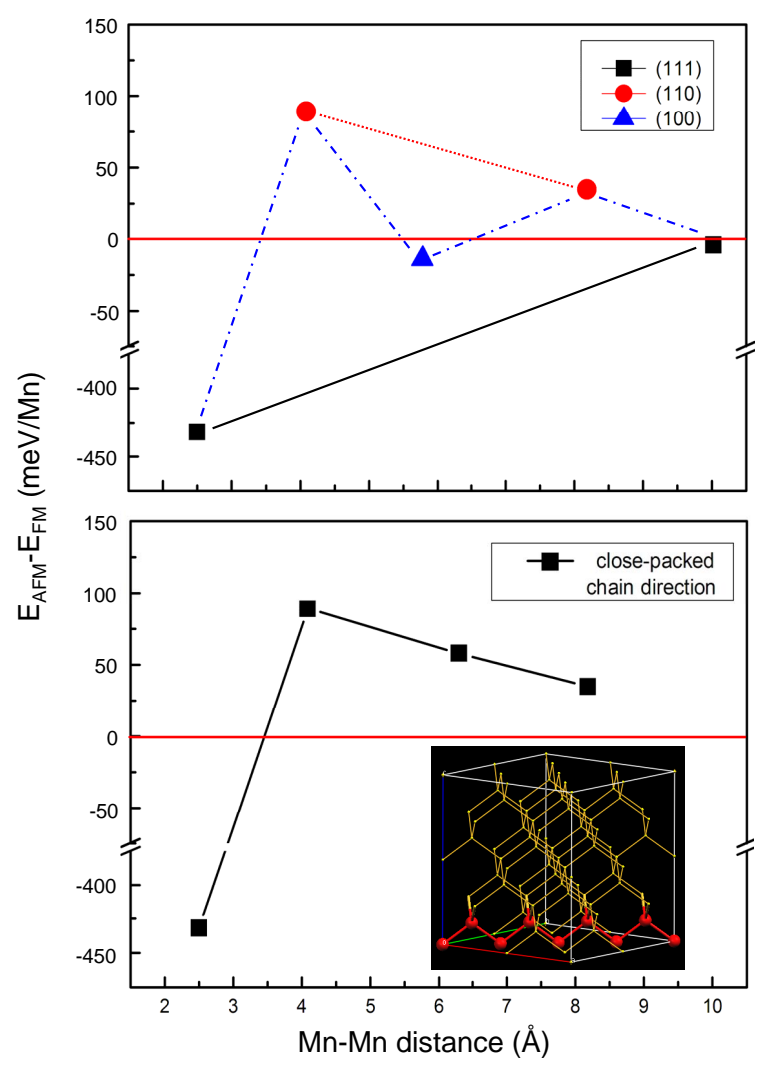

FIG. 10: (color online) Magnetic coupling along different crystal directions. Black squares are for the (111) direction, red dots for the (110) direction and blue triangles for the (100) direction. Magnetic coupling along a close-packed chain is illustrated in the inset.

a close-packed atom chain, which also shows monotonic decrease with distance except for the nearest neighbor value. This finding leads us to suggest that Mn ions in Ge are magnetically coupled through some paths consisting of covalently-bonded Ge atoms, a hypothesis which deserves to be checked by more detailed investigations.

There are two other important issues revealed in Fig. 9. First, the dispersive values of $\Delta E$ at a given distance in the co-doped case show a new kind of magnetic anisotropy. This anisotropy is conceptually different from the magnetic anisotropy that is typically discussed in the literature. The traditional definition refers to the cases in which the magnetization of a system exhibits anisotropy when the magnetic moment is polarized along different crystalline directions ${ }^{48}$, or when the coupling between two magnetic dopants is anisotropic depending on their relative orientation in the host material39. In contrast, here the two magnetic dopants are fixed in space, and the magnetic anisotropy is caused by the relative positions of the two $n$-type e-dopants surrounding the magnetic impurities. Second, from the presence of the $n$-type dopant, the FM interaction between two magnetic atoms on the whole preserves its magnitude rather than being substantially weakened. Thus, the influence of e-dopants on the magnetic properties of the whole system is not simply a weakening of the magnetic coupling by decreasing the number of interaction mediators.

Deeper understanding of the above observations requires a careful examination of the microscopic coupling mechanism. To this end, we consider three representative configurations, all with the same Mn-Mn separation fixed, equal to the next nearest neighbor distance in the Ge matrix: (a), a Mn-Mn pair in pure Ge as the reference structure; (b) and (c), a Mn/e-dopant-Mn/e-dopant pair with the strongest and weakest magnetic couplings, respectively. For the reference case shown in Fig. 11(a), the two Mn atoms share a Ge atom as their nearest neighbor. When the Mn pair is ferromagnetically coupled, the spin density in the plane containing the two Mn atoms and their mutual Ge neighbor (the $\left(\begin{array}{lll}1 & \overline{1} & 0\end{array}\right)$ plane indicated in Fig. 11(a)) is plotted in Fig. 11(b). The red (blue) area represents spin up (down) density. The large local magnetic moments of Mn induce spin polarization on the nearby non-magnetic Ge atoms, which are antiferromagnetically coupled with the Mn atoms.

The corresponding plots for case (b) are shown in Fig. 11(c) and Fig. 11(d). In this case, the two As atoms are not in the ( $\left.\begin{array}{lll}1 & 1 & 0\end{array}\right)$ plane, and the two Mn atoms still have the same Ge atom as their mutual nearest neighbor. Furthermore, the local magnetic moment of the bridging Ge atom shows little change, indicating that $J_{\text {eff }}$ essentially stays the same. To show the effect of As doping, we plot in Fig. 11(f) the spin density on the plane containing the two $\overline{\mathrm{Mn}}$ and one As atom (the (1 1 1 2) plane in Fig. 11(e)). Here, As acts as a donor helping to compensate the holes introduced by its neighboring $\mathrm{Mn}$, resulting in an increased local magnetic moment on each Mn atom, $S_{M n}\left(3.60 \mu_{B} \rightarrow 4.00 \mu_{B}\right)$. Therefore, the overall magnetic coupling between the two Mn atoms is enhanced relative to the pure Ge case.

For case (c), the corresponding plots are shown in Fig. 11(g) and Fig. 11(h). In this case, the two As and

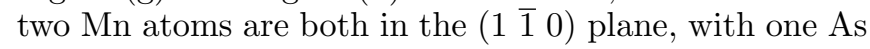
replacing the mutual nearest Ge neighbor of the two Mn atoms. Similar to case (b), here both $S_{M n 1}$ and $S_{M n 2}$ are also increased $\left(S_{M n 1}: 3.60 \mu_{B} \rightarrow 3.87 \mu_{B} ; S_{M n 2}: 3.60 \mu_{B} \rightarrow\right.$ $4.02 \mu_{B}$; the asymmetry in the increase is caused by the asymmetric locations of the two As atoms). However, because the local magnetic moment of the bridging atom is substantially decreased from that of case (a) $\left(\mathrm{Ge}:-0.16 \mu_{B} \rightarrow \mathrm{As}:-0.05 \mu_{B}\right)$, the corresponding $J_{\text {eff }}$ is also significantly weakened, leading to an overall weakened magnetic coupling between the two Mn atoms relative to the pure Ge case.

Summarizing, As as $n$-type dopant can enhance the local magnetic moments of neighboring Mn atoms, but itself is weakly spin polarized (much weaker than Ge). Therefore, if As serves as the bridging atom between two Mn atoms, the global magnetic coupling will be weak- 


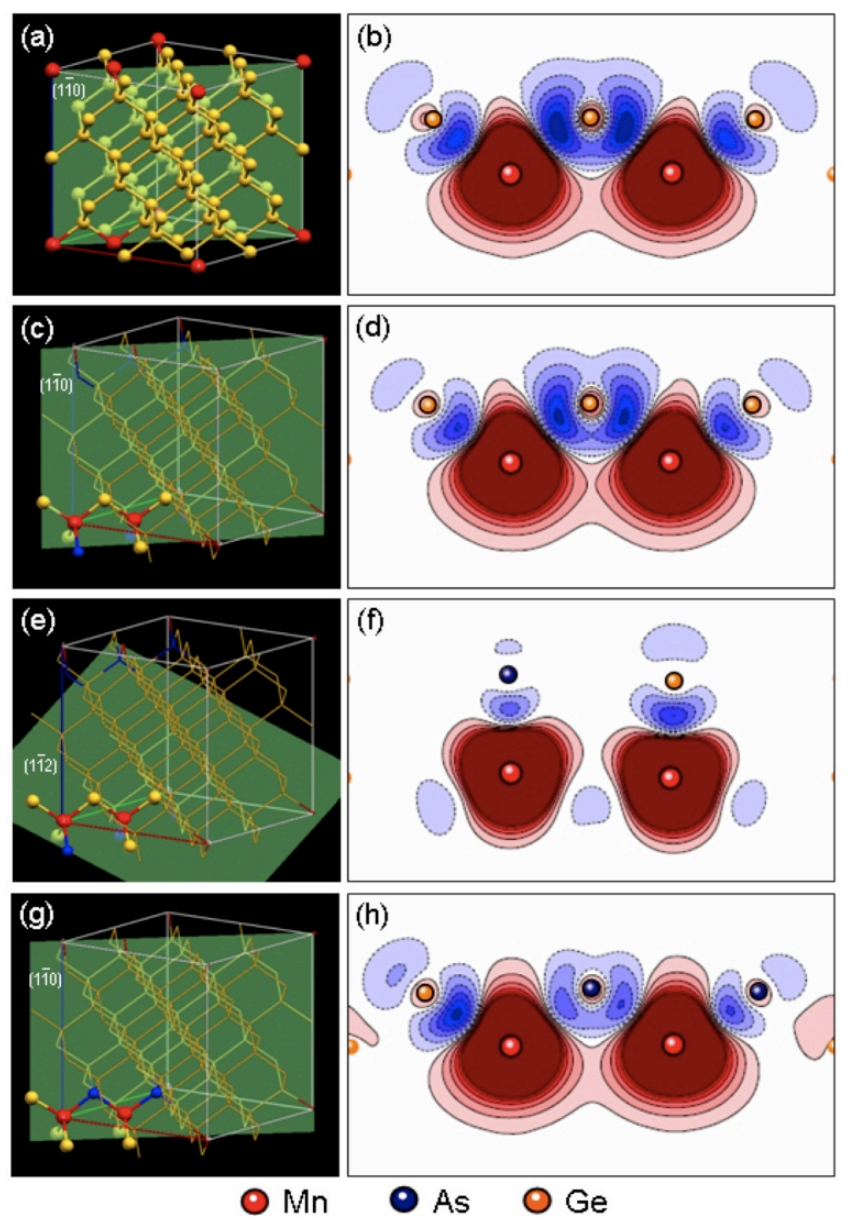

FIG. 11: (color online) The atomic structures and spin density plots of three representative configurations of two Mn TOMS, (a) and (b), and two Mn/As pairs, (c)-(h), doped Ge. In all structures, the two Mn atoms are fixed at the next nearest neighbor distance. The spin density plots are taken on the green plane as depicted in the structures on the left. The red and blue contours represent the two different spin components. (c) and (e) correspond to the configuration with the strongest magnetic coupling between two Mn ions, and (g) the weakest magnetic coupling.

ened. If As is located so as to only enhance the magnetic moment of Mn, with a Ge atom still bridging the Mn-Mn coupling, then the global magnetic coupling will be enhanced. This conclusion is further confirmed by checking other Mn-Mn distances.

\section{CURIE TEMPERATURE}

To study the macroscopic magnetic properties of the co-doped DMS materials using our ab initio results, we turn to the classical Heisenberg model:

$$
H=-\sum_{i, j} J_{i j} \sigma_{i} \cdot \sigma_{j}
$$

where $J_{i j}$ is the magnetic coupling constant between moment $i$ and $j$, and $\sigma_{i}$ is a unit vector representing the direction of spin $i$. Then the AFM-FM energy difference $\Delta E$ calculated in previous section is given by:

$$
\Delta E=E_{A F M}-E_{F M}=4 J_{12},
$$

with 1 and 2 the indices of the two moments in the supercell. With given coupling parameters, we then use Monte Carlo simulations to address the statistical mechanics of the DMS systems at finite temperatures. To eliminate finite size effects, the cumulant crossing method $\frac{40}{4 s}$ used to determine the Curie temperature. This two-step approach has the distinct advantage over the ordinary mean field approach, that both disorder and percolation effects are naturally and precisely taken into account $\underline{49150}$.

A subtle issue in the present case is the following: Since real interactions between magnetic atoms in DMS have a built-in multiatom nature, in an optimal Heisenberg description the coupling parameters must depend on the system geometry. This is difficult, if not entirely impossible $e^{51 / 52}$ to address, because of the very large number of possible configurations in a macroscopic system and some approximations are necessary.

The supercell ab initio approach 53 154/55156/57 employed here assumes only one approximation, the pairsuperposition approximation, which means that the interaction is exclusively pairwise and can be added independently to get the total interaction. Though this may not hold at high concentrations of magnetic moments, we claim that it should be a reasonable approximation at the low concentrations we considered (3.13\%-6\%), where the average distance between two $\mathrm{Mn}$ atoms, calculated by

$$
\bar{d}=2 \sqrt[3]{\frac{3}{32 \pi x}} a
$$

with $x$ the concentration and $a$ the lattice constant, ranges from $1.97 a$ to $1.58 a$, or $11.39 \AA$ to $9.14 \AA$ in the case of Mn doped Ge. Considering the bond length $d_{\text {bond }}$ in Ge is only about $2.5 \AA$, a separation $\sim 4 d_{\text {bond }}$ is large enough for the system to be treated in this approximation.

Using the ab initio coupling parameters for $\mathrm{Mn}_{x} \mathrm{Ge}_{1-x}$, we first find that $\mathrm{MC}$ does not yield identifiable $\mathrm{T}_{c}$ up to $x=6 \%$ (see below). Nevertheless, after co-doping with As, MC shows that the system has high $\mathrm{T}_{c}$, as summarized in Fig. 12, in which we also include the results from the mean field approximation (MFA) using the formula ${ }^{58}$

$$
T_{c}=\frac{1}{k_{B}} \cdot \frac{2 x}{3} \sum_{i \neq 0} J_{0 i} .
$$

These results show that the MFA greatly overestimates the Curie temperature, as established before 49150158 . At $x=5 \%, \mathrm{~T}_{c}$ is evaluated to be $264 \mathrm{~K}$ through $\mathrm{MC}$, which is much higher than the $118 \mathrm{~K}$ of $5 \% \mathrm{Mn}$ doped GaAs 59 . At the $6 \% \mathrm{Mn}$ concentration, $\mathrm{MC}$ gives a $\mathrm{T}_{c}$ higher than room temperature. Considering that $x=6 \%$ is already a 


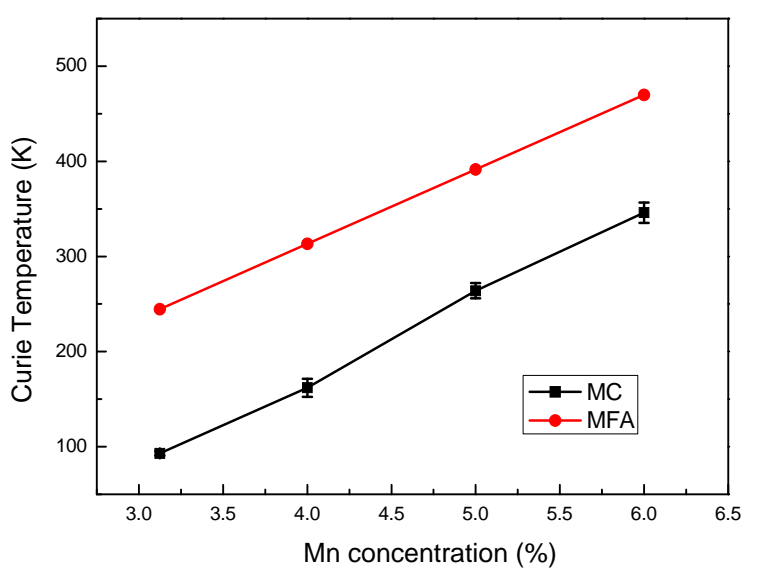

FIG. 12: (color online) Comparison between Curie temperatures calculated by the Monte Carlo approach (MC, black squares) and those obtained by the mean field approximation (MFA, red dots).

relatively high concentration, we expect that the pairsuperposition approximation may not be valid in this case. Arsenic doping can still be expected to dramatically change the magnetic properties of Mn doped Ge, namely, from no finite $\mathrm{T}_{c}$ to a potentially high $\mathrm{T}_{c}$ DMS material.

The dependence of the Curie temperature on $\mathrm{Mn}$ concentration, as obtained from the MC results, is almost linear. This behavior is partly due to the pairsuperposition approximation we used, meaning that the strength of magnetic coupling does not depend on $\mathrm{Mn}$ concentration. The only influence of concentration on $\mathrm{T}_{c}$ is the average number of magnetic impurity atoms on each coordination shell. Thus, after the configurational average, we expect that the dependence of $\mathrm{T}_{c}$ on $x$ resembles the linear one obtained within the MFA (Eqn.77). Another reason for this linearity is that the concentrations we studied are higher than the magnetic percolation threshold of this system ${ }^{58}$.

The presence of AFM couplings and the absence of $T_{c}$ in the case of pure Mn doped Ge suggests the possibility of a spin-glass ground state for this system. Jaeger et al ${ }^{60}$ claimed that at low temperatures $\mathrm{Mn}_{x} \mathrm{Ge}_{1-x}$ exhibits spin-glass-like behavior and the critical temperature of the spin-glass phase transition is $12 \mathrm{~K}$ and $15 \mathrm{~K}$, for Mn concentrations $x=0.04$ and $x=0.2$, respectively. To examine whether this is the case, we first study the spin-spin correlation function of $5 \% \mathrm{Mn}$ doped Ge, at $T=0.01 K$. The result is shown in Fig. 13, along with a plot for Mn/As co-doped Ge, for comparison. The correlation function of $\mathrm{Mn}_{x} \mathrm{Ge}_{1-x}$ decays very fast with increasing distance and approaches to zero, indicating the absence of FM order even at low temperatures. Using

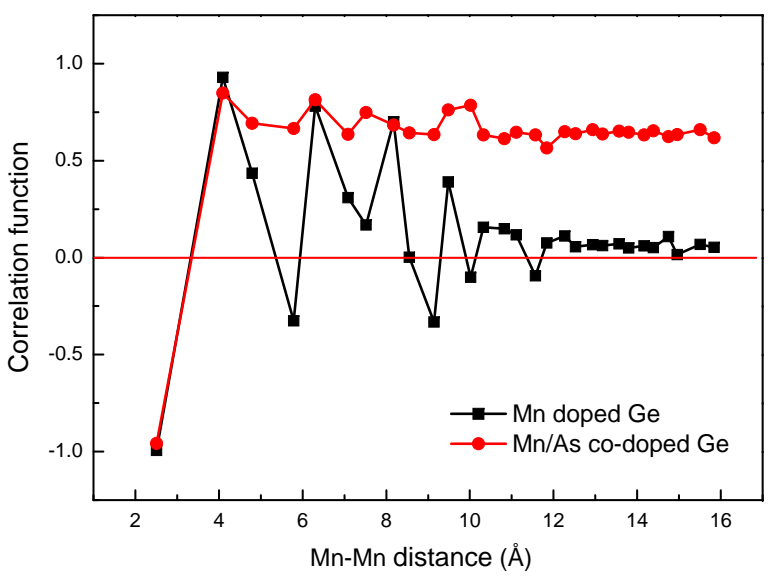

FIG. 13: (color online) Spin-spin correlation function obtained from the Monte Carlo simulations.

the spin-glass order parameter, defined as

$$
q=\frac{1}{N} \sum_{i}\left\langle\mathbf{S}_{i}\right\rangle^{2}
$$

and a similar cumulant crossing method $\sqrt{40}$ yields a transition temperature $\sim 5 \mathrm{~K}$, a value in semi-quantitative agreement with the results of Jaeger et al.60.

\section{DISCUSSION}

$\mathrm{Mn}_{x} \mathrm{Ge}_{1-x}$ has been attractive within the DMS community because of its easy incorporation into the current semiconductor industry. The mechanism of valence hole mediated ferromagnetism for $(\mathrm{Ga}, \mathrm{Mn})$ As was proposed years agd 4 446161 and has been extensively accepted ever since, but there is still no definitive theory for $\mathrm{Mn}_{x} \mathrm{Ge}_{1-x}$. points of this paper, is the difficulty of decreasing the percentage of interstitial Mn dopants. The other important point is the hard-to-control inhomogeneity of this system, which has been realized only in recent years. The high Curie temperature formerly reported in $\mathrm{Mn}_{x} \mathrm{Ge}_{1-x^{962}}$ is now thought to be due to the formation of $\mathrm{Mn}$ rich regions in the host semiconductor ${ }^{6134|60| 63 \mid 64}$. For example, Mn-rich nanodots ${ }^{65}$ and nanocolumns ${ }^{24 \mid 66 / 67}$ in $\mathrm{Mn}_{x} \mathrm{Ge}_{1-x}$ have been reported by many experimental groups, and later reproduced in Monte Carlo simulations 68 .

Despite the seemingly unavoidable precipitation or spinodal decomposition ${ }^{64}$ present during the growth of $\mathrm{Mn}_{x} \mathrm{Ge}_{1-x}$ samples, the study on homogeneously doped $\mathrm{Mn}_{x} \mathrm{Ge}_{1-x}$ has never stopped. Work by $\mathrm{Li}$ et al ${ }^{3463}$ indicates that the long-range $\mathrm{FM}$ order in $\mathrm{Mn}_{x} \mathrm{Ge}_{1-x}$ only exists at low temperatures $(\leq 12 \mathrm{~K})$. Jaeger et al ${ }^{60}$ claimed that even at low temperatures $\mathrm{Mn}_{x} \mathrm{Ge}_{1-x}$ shows 
spin-glass-like behavior, and proposed that this is due to the intercluster frustration between FM Mn-rich clusters. Recently, Zeng et al.15, using a newly developed subsurfactant epitaxy method, successfully grew clusterfree $\mathrm{Mn}_{x} \mathrm{Ge}_{1-x}$ samples with a Mn doping level of $0.25 \%$. Surprisingly, this low doping level (by normal DMS standards, where $1 \%$ to $5 \%$ is typical) led to a Curie temperature as high as over $400 \mathrm{~K}$.

The results in the present work provide a viewpoint that may resolve the seemingly conflicting experimental results discussed above. Specifically, we showed that the magnetic coupling between $\mathrm{Mn}$ ions in $\mathrm{Mn}_{x} \mathrm{Ge}_{1-x}$ oscillates between FM and AFM with increasing Mn-Mn distance and that homogeneous $\mathrm{Mn}_{x} \mathrm{Ge}_{1-x}$ exhibits spin glass behavior. Thus, the FM order observed in experiments could be due to spatially ordered structures, which are formed due to precipitation or spinodal decomposition. The high transition temperatures are expected because of the large concentration of magnetic moments within the clusters. On the other hand, the AFM frustration in this case only manifest itself in the inter-cluster interaction, and thus leads to the spin-glass behavior at low temperatures.

The unexpected high $\mathrm{T}_{c}$ in Zeng's work requires more discussion. Upon co-doping with $n$-type dopant As, the AFM coupling between Mn ions is absent, and a high Curie temperature emerges. We thus speculate that the high transition temperature in this case originates from this co-doping effect, and the unexpected $n$-type e-dopant here is most probably oxygen. Indeed, a recent study on the role of oxygen defects in $\mathrm{Mn}_{x} \mathrm{Ge}_{1-x}$ by Continenza and Profeta ${ }^{69}$ supports this scenario, namely that oxygen acts as an $n$-type dopant and facilitates the substitutional Mn doping. It is also reasonable to expect a positive influence of oxygen on the Mn-Mn magnetic coupling, which, together with the possible existence of Mn-rich regions, can lead to a high Curie temperature.

Recently, the works of Kuroda ${ }^{70}$ and Bonanni ${ }^{71}$ demonstrated experimentally that the aggregation of magnetic ions in DMS systems can be controlled by modifying the charge states of the magnetic dopants. This is in agreement with the spirit of our work, that is, charge states of impurity dopants play an important role in the growth kinetics of DMS materials and can lead to different structures with their own specific properties.

Finally, this work suggests that the enhancement of substitutional Mn concentration in group-IV DMS can be achieved in epitaxial growth by co-depositing with the e-dopants. More specifically, this co-doping method can be integrated in the recently developed subsurfactant epitaxial growth 15 , where pure Ge layers epitaxially grow on a $\mathrm{Ge}(100)$ substrate pre-covered with a submonolayer of Mn. During the growth process, the Mn atoms tend to diffuse upward to the subsurface layer, as predicted in a previous theoretical study ${ }^{72}$. When the growth is slow enough, a small fraction of the Mn atoms can be trapped in substitutional sites, which leads to homogeneous substitutional Mn doping with all the interstitial
Mn floating at the subsurface layer. However, the resulting $\mathrm{Mn}$ concentration is still pretty low (0.25\%). Here we propose that by co-depositing Ge with another $n$-type edopant, with very low depositing rates, the growth front could mimic the subsurfactant growth mode, but with more efficient substitutional trapping of $\mathrm{Mn}$. The Mn trapping rate can be controlled by changing the concentration of the e-dopant. Experimental confirmation of this co-doping scheme is highly desirable.

\section{CONCLUSION}

In conclusion, our ab initio DFT calculations show that in DMS materials additional $n$-type electronic dopants can serve to enhance the substitutional doping of $p$-type magnetic dopants such as Mn in the host group IV semiconductors Si and Ge. The additional dopants suppress to a large extent the charge and magnetic-moment compensating effects from interstitial Mn, which is detrimental to FM order. We calculate the magnetic coupling between moments associated with Mn atoms using the energy difference between parallel and antiparallel aligned pairs of Mn moments. We examined the unconventional magnetic anisotropy in $\mathrm{Mn} / \mathrm{As}$ co-doped Ge, namely, the dependence of magnetic coupling on the relative positions of magnetic ions and their neighboring assistant dopants. We find that the coupling oscillates between ferromagnetic (FM) and antiferromagnetic (AFM) with increasnig Mn-Mn distance in the Mn-doped Ge, whereas in As/Mn $n$ - $p$ co-doped Ge the coupling values at MnMn separations up to the 12 th coordination shell are all FM, except for the nearest-neighbor one. Moreover, we find that the FM-AFM oscillatory behavior in $\mathrm{Mn}_{x} \mathrm{Ge}_{1-x}$ is due to anisotropy rather than being the result of a RKKY-type interaction. Our Monte Carlo simulations, using magnetic coupling parameters obtained from the $a b$ initio calculations, indicate a high Curie temperature in $\mathrm{Mn} / \mathrm{As}-\mathrm{Ge}$ of $264 \mathrm{~K}$ at $5 \% \mathrm{Mn}$ doping. On the other hand, no FM order is observed in $\mathrm{Mn}_{x} \mathrm{Ge}_{1-x}$ (without co-doping) as $\mathrm{Mn}$ concentration ranges from $3.13 \%$ to $6 \%$. Thus, the homogeneously doped $\mathrm{Mn}_{x} \mathrm{Ge}_{1-x}$ is most likely a generic spin glass, with a spin-glass transition temperature of $5 \mathrm{~K}$ at $5 \%$ doping, also obtained from our Monte Carlo simulations. Accordingly, we suggest that the high Curie temperature observed experimentally in $\mathrm{Mn}_{x} \mathrm{Ge}_{1-x}$ is either due to the formation of Mn-rich spatially ordered regions, or to $n$ - $p$ co-doping effects from the $n$-type oxygen impurities, or a combination of both.

\section{Acknowledgments}

The authors thank Dr. Rong Yu for helpful discussions and Dr. Kirk H. Bevan for a critical reading of the manuscript. This work was supported in part by NSF grant Nos. DMR-0325218 and DMR-0606485, by DOE grant No. DE-FG02-05ER46209, and in part by the Di- 
vision of Materials Sciences and Engineering, Office of Basic Energy Sciences, DOE. The calculations were per- formed at NERSC of DOE and NCCS of ORNL.
1 S. A. Wolf et al., Science 294, 1488 (2001).

2 I. Žutić, J. Fabian, and S. Das Sarma, Rev. Mod. Phys. 76, 323 (2004).

${ }^{3}$ H. Ohno, Science 281, 951 (1998).

4 T. Dietl, H. Ohno, F. Matsukura, J. Cibert, and D. Ferrand, Science 287, 1019 (2000).

${ }^{5}$ R. N. Bhatt, M. Berciu, M. P. Kennett, and X. Wan, J. Supercond. 15, 71 (2002).

6 T. Jungwirth, J. Sinova, J. Mašek, J. Kučera, and A. H. MacDonald, Rev. Mod. Phys. 78, 809 (2006).

7 C. Timm, J. Phys.: Condens. Matter 15, R1865 (2003).

8 S. Sanvito, G. Theurich, and N. A. Hill, J. Supercond. 15, 85 (2002).

9 Y. D. Park et al., Science 295, 651 (2002).

10 H. Ohta, S. Okubo, J. Yoshikawa, Y. Nakashima, C. Urakawa, H. Nakayama, and T. Nishino, Physica B 298, 449 (2001).

11 H. Nakayama, H. Ohta, and E. Kulatov, Physica B 302, 419 (2001).

12 S. Abe, Y. Nakasima, S. Okubo, H. Nakayama, T. Nishino, H. Yanagi, H. Ohta, and S. Iida, Appl. Surf. Sci. 142, 537 (1999).

13 M. Bolduc, C. Awo-Affouda, A. Stollenwerk, M. B. Huang, F. G. Ramos, G. Agnello, and V. P. LaBella, Phys. Rev. B 71, 033302 (2005).

14 S. Picozzi, F. Antoniella, A. Continenza, A. MoscaConte, A. Debernardi, and M. Peressi, Phys. Rev. B 70, 165205 (2004).

15 C. Zeng, Z. Y. Zhang, K. van Benthem, M. F. Chisholm, and H. H. Weitering, Phys. Rev. Lett. 100, 066101 (2008).

16 S. C. Erwin and A. G. Petukhov, Phys. Rev. Lett. 89, 227201 (2002).

17 R. Wu, Phys. Rev. Lett. 94, 207201 (2005).

18 S. Hao and Z. Y. Zhang, Phys. Rev. Lett. 99, 166101 (2007).

19 K. M. Yu, W. Walukiewicz, T. Wojtowicz, I. Kuryliszyn, X. Liu, Y. Sasaki, and J. K. Furdyna, Phys. Rev. B 65, 201303(R) (2002).

${ }^{20}$ K. C. Ku et al., Appl. Phys. Lett. 82, 2302 (2003).

21 K. W. Edmonds et al., Phys. Rev. Lett. 92, 037201 (2004).

22 F. D'Orazio et al., J. Magn. Magn. Mater. 262, 158 (2003).

23 C. G. Zeng, W. Zhu, S. C. Erwin, Z. Y. Zhang, and H. H. Weitering, Phys. Rev. B 70, 205340 (2004).

24 M. Jamet et al., Nat. Mater. 5, 653 (2006).

25 Y. H. Kwon, T. W. Kang, H. Y. Cho, and T. W. Kim, Solid State Commun. 136, 257 (2005).

${ }^{26}$ W. Zhu, Z. Y. Zhang, and E. Kaxiras, Phys. Rev. Lett. 100, 027205 (2008).

27 G. Kresse and J. Furthmüller, Phys. Rev. B 54, 11169 (1996).

28 P. E. Blöchl, Phys. Rev. B 50, 17953 (1994).

29 G. Kresse and D. Joubert, Phys. Rev. B 59, 1758 (1999).

30 J. P. Perdew, K. Burke, and M. Ernzerhof, Phys. Rev. Lett. 77, 3865 (1996).

31 C. R. Hubbard, H. E. Swanson, and F. A. Mauer, J. Appl. Crystallogr. 8, 45 (1975).

32 H. P. Singh, Acta Crystallogr. 24A, 469 (1968).
${ }^{33}$ G. Henkelman, B. P. Uberuaga, and H. Jónsson, J. Chem. Phys. 113, 9901 (2000).

34 A. P. Li, J. Shen, J. R. Thompson, and H. H. Weitering, Appl. Phys. Lett. 86, 152507 (2005).

35 S. B. Ma et al., Solid State Commun. 140, 192 (2006).

36 Y.-J. Zhao, T. Shishidou, and A. J. Freeman, Phys. Rev. Lett. 90, 047204 (2003).

37 A. Stroppa, S. Picozzi, A. Continenza, and A. J. Freeman, Phys. Rev. B 68, 155203 (2003).

38 H. Weng and J. Dong, Phys. Rev. B 71, 035201 (2005).

39 P. Mahadevan, A. Zunger, and D. D. Sarma, Phys. Rev. Lett. 93, 177201 (2004).

40 K. Binder and D. W. Heermann, Monte Carlo Simulation in Statistical Physics (Springer, Berlin, 2002).

${ }^{41}$ K. Binder, Phys. Rev. Lett. 47, 693 (1981).

42 H. Ohno, et al., Appl. Phys. Lett. 69, 363 (1996).

43 H. Ohno, F. Matsukura, T. Omiya, and N. Akiba, J. App. Phys. 85, 4277 (1999).

44 A. J. R. da Silva, A. Fazzio, and A. Antonelli, Phys. Rev. B 70, 193205 (2004).

45 S. Picozzi, M. Ležaić, and S. Blügel, Phys. Stat. Sol. (a) 203, 2738 (2006).

46 F. Matsukura, H. Ohno, A. Shen, and Y. Sugawara, Phys. Rev. B 57, R2037 (1998).

47 J. Kudrnovský, I. Turek, V. Drchal, F. Máca, P. Weinberger, and P. Bruno, Phys. Rev. B 69, 115208 (2004).

48 D. S. Wang, R. Wu, and A. J. Freeman, Phys. Rev. Lett. 70, 869 (1993)

49 K. Sato, W. Schweika, P. H. Dederichs, and H. KatayamaYoshida, Phys. Rev. B 70, 201202(R) (2004).

${ }^{50}$ L. Bergqvist, O. Eriksson, J. Kudrnovský, V. Drchal, P. A. Korzhavyi, and I. Turek, Phys. Rev. Lett. 93, 137202 (2004).

51 A. Franceschetti, S. V. Dudiy, S. V. Barabash, A. Zunger, J. $\mathrm{Xu}$, and M. van Schilfgaarde, Phys. Rev. Lett. 97, 047202 (2006).

52 A. Franceschetti, A. Zunger, and M. van Schilfgaarde, J. Phys.: Condens. Matter 19, 242203 (2007).

53 S. Sanvito and N. A. Hill, Appl. Phys. Lett. 78, 3493 (2001).

54 L. M. Sandratskii and P. Bruno, Phys. Rev. B 67, 214402 (2003).

55 A. J. R. da Silva et al., J. Phys. Condens. Matter 16, 8243 (2004).

56 M. Wierzbowska, D. Sánchez-Portal, S. Sanvito, Phys. Rev. B 70, 235209 (2004).

57 S. Picozzi and M. Ležaić, New J. Phys. 10, 055017 (2008).

58 L. Bergqvist, O. Eriksson, J. Kudrnovský, V. Drchal, A. Bergman, L. Nordström, and I. Turek, Phys. Rev. B 72, 195210 (2005).

59 K. W. Edmonds, K. Y. Wang, R. P. Campion, A. C. Neumann, N. R. S. Farley, B. L. Gallagher, and C. T. Foxon, Appl. Phys. Lett. 81, 4991 (2002).

60 C. Jaeger et al., Phys. Rev. B 74, 045330 (2006).

61 H. Ohno, J. Magn. Magn. Mater. 200, 110 (1999).

62 S. Cho et al., Phys. Rev. B 66, 033303 (2002).

63 A. P. Li, J. F. Wendelken, J. Shen, L. C. Feldman, J. R. 
Thompson, and H. H. Weitering, Phys. Rev. B 72, 195205 (2005).

64 T. Dietl, J. Appl. Phys. 103, 07D111 (2008).

65 D. Bougeard, S. Ahlers, A. Trampert, N. Sircar, and G. Abstreiter, Phys. Rev. Lett. 97, 237202 (2006).

66 J.-S. Kang et al., Phys. Rev. Lett. 94, 147202 (2005).

${ }^{67}$ A. P. Li et al., Phys. Rev. B 75, 201201(R) (2007).

${ }^{68}$ H. Katayama-Yoshida et al., Phys. Status Solidi A 204, 15
(2007)

69 A. Continenza and G. Profeta, Phys. Rev. B 78, 085215 (2008).

70 S. Kuroda et al., Nature Materials 6, 440 (2007).

71 A. Bonanni et al., Phys. Rev. Lett. 101, 135502 (2008).

${ }^{72}$ W. Zhu, H. H. Weitering, E. G. Wang, E. Kaxiras, and Z. Y. Zhang, Phys. Rev. Lett. 93, 126102 (2004). 